\title{
Médiévales
}

Langues, Textes, Histoire

65 | automne 2013

Le couple dans le monde franc

\section{Le couple aristocratique au haut Moyen Âge}

The Aristocratic Couple in the Early Middle Ages

\section{Régine Le Jan}

\section{(2) OpenEdition}

Journals

Édition électronique

URL : https://journals.openedition.org/medievales/7083

DOI : $10.4000 /$ medievales.7083

ISSN : 1777-5892

Éditeur

Presses universitaires de Vincennes

Édition imprimée

Date de publication : 1 décembre 2013

Pagination : 33-46

ISBN : 978-2-84292-396-9

ISSN : 0751-2708

\section{Référence électronique}

Régine Le Jan, "Le couple aristocratique au haut Moyen Âge », Médiévales [En ligne], 65 | automne 2013, mis en ligne le 20 janvier 2014, consulté le 22 avril 2022. URL : http://journals.openedition.org/ medievales/7083 ; DOI : https://doi.org/10.4000/medievales.7083 
Régine LE JAN

\section{LE COUPLE ARISTOCRATIQUE AU HAUT MOYEN ÂGE}

Au III ${ }^{\mathrm{e}}$ siècle, le juriste Modestin définissait le mariage comme «l'union de l'homme et de la femme, une communauté de toute une vie, la mise en commun de ce qui relève du droit humain et du droit divin ${ }^{1}{ }$, établissant ainsi le couple comme coextensif du mariage. À la fin de l'Antiquité, le développement d'une parenté de plus en plus cognatique et le christianisme avaient affaibli les vieilles gentes et les élites romaines mettaient en avant la conjugalité. En témoignent les inscriptions funéraires qui la rendent visible, ou encore le traité Ad Gregoriam in palatio, rédigé probablement à la fin du $\mathrm{v}^{\mathrm{e}}$ siècle par un certain Jean pour une Romaine de rang sénatorial qui était mariée. L'auteur y développe les devoirs d'une domina qui participe directement, en tant qu'épouse chrétienne, à la gestion conjugale ${ }^{2}$. En système de parenté cognatique, le mariage a une double fonction: non seulement il assure la reproduction de la lignée par la création d'une nouvelle cellule sociale, mais il établit aussi un lien d'amitié fort entre donneur et preneur de femme. Nous savons qu'au haut Moyen Âge, le mariage était un élément essentiel des stratégies distinctives développées par les élites dirigeantes pour se maintenir et se reproduire en tant qu'élites ${ }^{3}$. Mais pour autant, tous les mariages atteignaient-ils ce but? Est-il si sûr que les couples aient toujours été assez

1. Cité par J. Gaudemet, Le Mariage en Occident : les mours et le droit, Paris, 1987, p. 15.

2. K. CoOper, The Fall of the Roman Household, Cambridge, 2007, trad. anglaise du texte, p. 239-283.

3. R. LE JAN, Famille et pouvoir dans le monde franc (VII $-I X^{e}$ siècle). Essai d'anthropologie sociale, Paris, 1995, p. 288-332. Voir entre autres les exemples donnés par P. DEPREUX, «L'intégration des élites aristocratiques de Bavière et de Saxe au royaume des Francs. Crise ou opportunité ?», dans F. Bougard, L. Feller, R. Le JAn éd., Les Élites au haut Moyen Âge. Crises et renouvellements, Turnhout, 2006, p. 225-252. 
solides pour développer les stratégies familiales et les promesses mises en eux ? Il s'agit d'une question importante, qui relève moins du droit que des forces de collaboration dans le couple, des modalités d'officialisation et de l'identité du couple en tant qu'acteur social. J'ai privilégié la longue durée pour mettre en lumière les changements et discuter l'importance du moment carolingien. Dans les années 820 arrive en effet à maturité une théorie politique, formulée par les évêques, qui repose sur une conception familiale du pouvoir: l'empire est conçu comme une famille, irriguée par l'amour qui s'épanouit dans la fidélité. Inscrite dans un ordre qui fait du couple et de la famille impériale l'atome et le modèle sur lequel se construit l'édifice social, l'idéologie carolingienne confère au système politique une affectivité performative. Il convient donc de voir si le $\mathrm{IX}^{\mathrm{e}}$ siècle représente une césure dans l'histoire du couple aristocratique. Y a-t-il un avant et un après $\mathrm{IX}^{\mathrm{e}}$ siècle?

\section{Plusieurs femmes pour un seul homme?}

Les travaux en cours sur la masculinité, appliqués à l'échelle du globe, soulignent à quel point les modèles familiaux sont variés. Certes, au haut Moyen Âge, le mode de résidence virilocal, qui semble avoir été le mode le plus fréquent, avait pour effet que la femme venait habiter dans la maison de son mari, ce qui contribuait à bien identifier le couple. Mais il y a eu aussi des cas de résidence patrilocale, où des jeunes ménages vivaient dans la maison des parents plus âgés de l'un ou l'autre conjoint, ce qui diminuait l'autonomie et la visibilité des couples.

Cela étant, la famille conjugale, qui est un héritage de l'Antiquité tardive, a toujours été le seul modèle promu par l'Église. Saint Augustin a énoncé les biens du mariage au $\mathrm{IV}^{\mathrm{e}}$ siècle et les vies de saints offrent un modèle d'organisation parentale strictement fondé sur le couple et ses enfants. Le voyage de saint Colomban en Neustrie au début du $\mathrm{VII}^{\mathrm{e}}$ siècle, tel que le décrit Jonas de Bobbio, est une série d'étapes amicales dans des familles chrétiennes systématiquement représentées comme des couples avec enfants. Les Vitae relatant l'arrivée des missionnaires anglo-saxons en Germanie au viII ${ }^{\mathrm{e}}$ siècle décrivent aussi des couples conjugaux, à qui les hommes de Dieu reprochent seulement leurs mariages trop proches. Mais nous savons à quel point le discours hagiographique est construit, et les hagiographes ont omis d'aborder de front la question de la sexualité pour s'en tenir au mariage légal. Car si l'attirance sexuelle et la sexualité féminine étaient des dangers pour la société médiévale, qui les percevait comme des facteurs de désordre, la sexualité masculine était considérée comme un facteur de distinction, qui pouvait contribuer à la reproduction sociale, à condition d'être régulée. Et les missionnaires, qui étaient avant tout pragmatiques, se sont contentés de promouvoir le mariage légal, de faire respecter les interdits 
en matière d'inceste et de condamner les rapts ${ }^{4}$, sans s'attaquer de front aux pratiques polygyniques, contre lesquelles ils étaient impuissants.

Dans les sociétés patriarcales, la polygynie, entendue comme le fait qu'un homme entretienne des liaisons durables et connues avec plusieurs femmes, est un moyen de tourner le poids des contraintes sur le mariage, de contrôler la sexualité, de parer aux dangers de la mortalité infantile, d'élargir les réseaux. Or, des études récentes, réalisées pour la période $\mathrm{du}_{\mathrm{IX}}{ }^{\mathrm{e}}-\mathrm{XIII}{ }^{\mathrm{e}}$ siècle à partir de la Scandinavie, de l'Irlande, de l'Espagne du Nord, tendent à montrer que la polygynie était un signe distinctif de l'aristocratie ${ }^{5}$. Dans le monde franc, il y a effectivement des signes indéniables de polygynie, successive ou simultanée ${ }^{6}$. Certains rois mérovingiens sont bien connus pour avoir été des polygames et, à la fin du vir siècle, le maire du palais Pépin II était marié à Plectrude depuis de longues années quand il prit pour épouse une noble et élégante jeune femme, dont il eut Charles; et ce même Charles Martel eut des enfants, parfaitement assumés, d'au moins trois femmes. Charlemagne eut successivement cinq épouses et, à la fin de sa vie, il eut au moins quatre concubines connues, et sans doute simultanées. Nous ne savons pas si Louis le Pieux avait renvoyé son épouse de jeunesse, dont il avait deux enfants, quand il épousa Ermengarde, mais Lothaire II a continué à avoir des relations suivies avec Waldrade, la mère de ses enfants, tout en étant marié à Theutberge. Charles le Simple a eu trois épouses successives et plusieurs liaisons, d'où sont issus des enfants, certes illégitimes, mais pourvus de bonnes positions. Le duc Hugues le Grand se maria quatre fois, sans compter des liaisons ; Hugues d'Arles était un polygame notoire et Robert le Pieux fut marié trois fois et divorça deux fois. On pourrait multiplier les exemples.

À la polygynie s'ajoutait une certaine instabilité des couples, car la situation de celles qui n'avaient pas d'enfants, ou encore de celles qui avaient cessé d'être intéressantes en termes d'alliance, devenait précaire. $\mathrm{Au} \mathrm{vI}$ siècle, l'assassinat de Galswinthe, épouse wisigothe du roi Chilpéric I ${ }^{\text {er }}$, est dû au moins autant à la mort de son père Athanagild, qui avait ôté son intérêt au mariage, qu'à la jalousie de Frédégonde. Plectrude n'avait donné que deux fils à Pépin II quand il prit une autre épouse, peut-être pour avoir d'autres fils, comme l'a suggéré

4. Sur le rapt, S. JoYe, La Femme ravie. Le mariage par rapt dans les sociétés occidentales du haut Moyen Âge, Turnhout, 2012.

5. M. BoRgolte, «Kulturelle Einheit und religiöse Differenz: Zur Verbreitung der Polygynie im mittelalterlichen Europa », Zeitschrift für historische Forschung, 31 (2004), p. 1-36; J. RüDIGER, Der König und seine Frauen. Polygynie und politische Kultur in Europa (9.-13. Jh.), Habilitationsschrift Humboldt-Universität Berlin, 2006.

6. P. StAFFord, Queens, Concubines and Dowagers, Athens (Géorgie), 1983, p. 62-79; R. LE JAN, Famille et pouvoir..., p. 271-277; A. EsmyoL, Geliebte oder Ehefrau? Konkubinen im frühen Mittelalter, Cologne, 2002. 
Brigitte Kasten ${ }^{7}$. Dans les années 830, le couple formé par Dhuoda et Bernard de Septimanie fut sans doute fragilisé par les accusations portées contre Bernard, mais probablement aussi par le fait que Dhuoda n'avait eu longtemps qu'un seul fils ${ }^{8}$. Et, au tournant de l'an Mil, le comte Foulques Nerra fut accusé d'avoir fait brûler son épouse Élisabeth pour s'en débarrasser.

La polygynie brouillait l'image du couple, si l'une des coépouses ne s'imposait pas sur les autres. Chez les Lombards, la loi y contribua à partir du moment où elle fit la différence entre les enfants légitimes et les enfants naturels, les premiers étant sans doute ceux de l'épouse légale ${ }^{9}$. Mais les choses n'étaient pas aussi claires dans le monde franc, où les enfants des différentes coépouses libres avaient des droits ${ }^{10}$. On a fait justice de la Friedelehe, ce mariage légal mais de second rang que les juristes allemands du XIX ${ }^{\mathrm{e}}$ siècle ont inventé pour clarifier ce qui est obscur, mais tout porte à croire qu'un homme a pu avoir plusieurs uxores $^{11}$, c'est-à-dire plusieurs épouses légitimes. Le fait est attesté pour les rois mérovingiens jusqu'à Dagobert, il l'est aussi pour Pépin II. Pour l'auteur anonyme du Liber Historiae Francorum, Plectrude est uxor nobilissima et sapientissima de Pépin II ${ }^{12}$, et Alpais est une alia uxor ${ }^{13}$. Elle est aussi définie comme une uxor par l'auteur de la continuation de Frédégaire ${ }^{14}$. Alpais a donc été une coépouse de Plectrude et les sources ne commencent à désigner la mère de Charles comme une concubine qu'au IX ${ }^{\mathrm{e}}$ siècle, dans le contexte des pressions de l'Église sur le mariage ${ }^{15}$. Pépin II eut encore d'une autre femme, dont nous ignorons le nom, au moins un fils, Childebrand, qui est plus jeune que Charles (et qui est l'auteur de la première continuation de Frédégaire $\left.{ }^{16}\right)$. Même chose pour Himiltrude, mère du fils aîné de Charlemagne, qui ne fut qualifiée de concubine

7. B. KASTEN, Königssöhne und Königsherrschaft. Untersuchungen zur Teilhabe am Reich in der Merowinger- und Karolingerzeit, Hanovre, 1997, p. 71.

8. R. LE JAN, «The Multiple Identities of Dhuoda», dans R. MCKITTERICK, R. Corradini éd., Ego Trouble in the Early Middle Ages. Authors and their Identities in the Early Middle Ages, Vienne, 2010, p. 211-220.

9. Edictum Rotharii (643), c. 153-164 (dans Le leggi dei Langobardi. Storia, memoria e diritto di un popolo germanico, éd. C. AzZARA et S. GASPARRI, Rome, 2005, p. 46-50). La distinction n'est pas explicitée par ailleurs, ni mise en relation avec le mariage.

10. B. KASTEN, Königssöhne..., p. 70-81.

11. J. КосH, «Karl Martel - Ein minderrechtleicher Erbe Pippins », dans J. JARNUT, U. NonN, M. Richter éd., Karl Martell in seiner Zeit, Sigmaringen, 1994, p. 150-169 (p. 151-152).

12. Liber Historiae Francorum, c. 48 (MGH SSRM II, p. 323).

13. Ibid., c. 49, p. 324F.

14. FrédÉGaire, Chronique des temps mérovingiens. Continuations, c. 6 (trad. O. Devillers et J. Meyers, Turnhout, 2006).

15. ERchembert, Breviarum Regum Francorum (éd. G. Pertz, MGH SSII, Hanovre, 1829, a. 715-827, p. 328); J. Косн, «Karl Martell...».

16. B. Kasten, Königssöhne..., p. 80-81. 
qu'au IX' siècle, dans la Vie de Charlemagne d'Éginhard. Charlemagne la conserva peut-être auprès de lui après son mariage avec la Lombarde et elle fut inhumée à Nivelles, l'abbaye familiale des Carolingiens. La compétition et les tensions, qui ne devaient pas manquer de se développer au sein des familles polygyniques entre les coépouses et entre les enfants issus de plusieurs lits, pouvaient affaiblir la visibilité du couple. Il y eut assurément une forte hostilité entre Plectrude et Alpais, qui valut peut-être à l'évêque Lambert de Liège, apparenté à Plectrude, d'être tué par Dodo, probablement frère d'Alpais ${ }^{17}$. Mais pour Pépin II, les deux épouses étaient aussi importantes l'une que l'autre, et si Plectrude finit par l'emporter, c'est en raison d'un capital social plus important, en termes de richesses, de prestige et de réseaux. Non seulement Pépin II ne divorça pas d'elle, mais il vivait avec Alpais depuis quelques années déjà et elle lui avait donné un fils quand, en 697, il fit avec Plectrude une donation à Saint-Arnoul de Metz ${ }^{18}$.

Pour les évêques, la question était de distinguer une épouse légitime des autres femmes. À partir du milieu du viII ${ }^{\mathrm{e}}$ siècle, ils ont donc cherché à imposer qu'elle soit régulièrement dotée et épousée selon des rites publics. Mais au début du IX ${ }^{\mathrm{e}}$ siècle, il n'était sans doute pas encore aisé de définir et de distinguer clairement une épouse légitime d'une concubine. Par la suite, les conciles du $\mathrm{IX}^{\mathrm{e}}$ siècle ont tenté d'imposer la monogamie, en interdisant d'avoir simultanément deux concubines ou une épouse et une concubine, toutes interdictions qui sont largement restées lettre morte. Néanmoins, la hiérarchisation de la société a renforcé la visibilité du couple conjugal aristocratique, même si l'épouse devait souvent admettre que son mari ait d'autres partenaires sexuelles ${ }^{19}$.

La polygynie, simultanée ou successive, a donc certainement été une pratique courante dans l'aristocratie du haut Moyen Âge, sous des formes variées. Elle n'a pas manqué de créer des tensions et de fragiliser les couples. Néanmoins, les rites de sociabilité et les stratégies matrimoniales complexes attestent de l'importance de l'épouse légitime. Demandons-nous donc si le couple développait des actions communes et coordonnées en matière de gestion du patrimoine, de stratégies mémoriales, de domination du groupe familial, ou si les actions étaient uniquement le fait du mari.

17. Une source certes tardive, la Vita Landiberti de Sigebert de Gembloux, raconte que le domesticus Dodo, frère d'Alpais, avait tué l'évêque de Liège Lambert en 705 parce que ce dernier avait reproché à Pépin II d'avoir pris sa sœur pour concubine («pro pellicatu suo ») : Vita Landiberti auctore Sigiberto, c. 16 (MGHSSRM VI, p. 398). Voir R. GERBERDING, The Rise of the Carolingians and the Liber Historiae Francorum, Oxford, 1987, p. 118-119 et P. Fouracre, The Age of Charles Martel, Londres, 2000, p. 55.

18. Die Urkunden der Arnulfinger, $\mathrm{n}^{\circ}$ 2, éd I. HeIDRICH, online http://www.uni-bonn.de/ uph202.

19. Sur la hiérarchisation sociale, voir F. Bougard et R. LE JAN, «Hiérarchie: le concept et son application dans les sociétés du haut Moyen Âge», dans F. Bougard, D. Iogna-Prat, R. Le Jan éd., Hiérarchie et stratification sociale dans l'Occident médiéval (400-1100), Turnhout, 2008. 


\section{Le couple et la gestion du patrimoine}

Même si la femme était considérée comme la maîtresse de maison, les données de l'archéologie contredisent l'idée d'une division "genrée» de l'espace, qui réserverait l'intérieur aux femmes et l'extérieur aux hommes. De la Scandinavie à la Gaule du Nord au moins, la maison était un espace neutre, où intervenaient aussi bien les hommes que les femmes ${ }^{20}$. Le couple disposait aussi d'un patrimoine commun, que symbolisait la remise à l'épouse d'ustensiles de ménage, mais aussi de bétail ou de terres données en pleine propriété ou sous forme d'usufruit des acquêts conjugaux (un tiers chez les Francs) ${ }^{21}$. Installés dans leur propre maison et possédant leurs propres biens, les époux devaient collaborer au travail et au maintien du patrimoine. Les possessions de chacun des époux étaient intégrées à la masse des biens conjugaux, qui étaient gérés par le mari, même si l'épouse conservait ses droits propres ${ }^{22}$. Cette tutelle maritale s'est probablement renforcée au très haut Moyen Âge avec la sédentarisation des populations, quand la terre est devenue l'élément le plus important du patrimoine aristocratique et que les familles se sont préoccupées du devenir de ce patrimoine en cherchant à contourner la dévolution divergente des biens. L'interdiction de transmettre la terre salique aux femmes ou les réserves mises à la possibilité qu'avaient les femmes lombardes de disposer seules de leurs biens en sont des indices ${ }^{23}$. Certes, les femmes avaient la capacité juridique de transférer leurs biens, mais les mentions d'achats, de ventes, d'échanges réalisés par des femmes seules sont rares et concernent surtout des veuves, alors que les hommes interviennent fréquemment seuls.

L'échange public des biens était un moyen pour le couple de développer des stratégies communes et de s'officialiser en tant qu'acteur social. De tels transferts sont attestés dès la fin du vi ${ }^{\mathrm{e}}$ siècle. On apprend ainsi dans le testament du fils d'Ida, daté par Josiane Barbier de 567-584, que le testateur avait acquis la villa Lectericus en Étampois par échange avec Hero et son épouse ${ }^{24}$. Cependant, dans le monde franc, la part des transferts réalisés par des couples reste minoritaire et stable durant la période $\mathrm{VIII}^{\mathrm{e}}-\mathrm{X}^{\mathrm{e}}$ siècle, oscillant autour de $15 \%$ de l'ensemble

20. S. Croix, Work and Space in Rural Settlements in Viking-Age Scandinavia - Gender Perspectives, Thèse de doctorat de l'université d'Aarhus, 2012.

21. Sur les dots, F. Bougard, L. Feller, R. Le JAn éd., Dots et douaires dans le haut Moyen Âge, Rome, 2002.

22. D. Hellmuth, Frau und Besitz. Zum Handlungsspielraum von Frauen in Alamannien (700940), Sigmaringen, 1998.

23. C. LA Rocca, «Pouvoirs des femmes, pouvoir de la loi dans l'Italie lombarde», dans S. LEBECQ et al. éd., Femmes et pouvoirs des femmes à Byzance et en Occident ( $V I^{e}-X I^{e}$ s.), Villeneuved'Ascq, 1999, p. 37-50.

24. Chartae latinae antiquiores (ChLa) : Facsimile-Edition of the Latin Charters Prior to the Ninth Century, 13, France I, no 569, éd. H. Atsma, J. Vezin, Dietikon, Zürich, 1981, p. 88. 
des transactions (à Saint-Gall, 505 donations faites par un homme, 72 par une femme, veuve le plus souvent, 64 par un couple ${ }^{25}$ ). Les couples intervenaient conjointement quand la donation concernait tout ou partie des biens de l'épouse. Notons cependant qu'en Bavière, les scribes, ayant rédigé après coup des notices, décrivent les donations comme des actes conjoints, réalisés par le couple ${ }^{26}$, ce qui suggère que les épouses pouvaient être présentes, même quand elles n'intervenaient pas directement dans l'acte de donation.

Les donations aux églises, et plus encore les fondations monastiques, qui permettaient de transformer du capital économique en capital symbolique, d'acquérir du prestige et du pouvoir, et aussi d'élargir leurs réseaux, étaient particulièrement importantes pour la visibilité du couple. En reprenant le cas emblématique de Pépin II, on constate qu'Alpais, quoique uxor, n'est jamais intervenue auprès de Pépin dans les affaires patrimoniales, alors que Plectrude a fondé avec son mari les monastères d'Echternach et de Süsteren sur ses propres domaines ${ }^{27}$. Le couple développait ainsi des stratégies patrimoniales collaboratives qui servaient la domination pippinide tout en renforçant la position de Plectrude. La diffusion des donations en précaire, avec réserve d'usufruit pour les deux époux, accentua la tendance générale du VIII ${ }^{\mathrm{e}}$ au $\mathrm{X}^{\mathrm{e}}$ siècle $^{28}$, surtout quand les biens en question provenaient exclusivement du patrimoine de l'épouse: en 795, le comte Alberic donna à sa fille des biens qu'avec son mari, le comte Gagenfrid, elle offrit ensuite au monastère de Prüm pour les tenir conjointement en précaire ${ }^{29}$.

Aux $x^{\mathrm{e}}$ et $\mathrm{XI}^{\mathrm{e}}$ siècles, la proportion de donations conjointes d'un couple ne varie guère, mais désormais le consentement de l'épouse est systématiquement requis, avec celui d'autres ayants droit, quand le mari fait une donation sur ses biens propres. Le fait n'est pas nouveau - on en trouve des exemples dès le VIII ${ }^{\mathrm{e}}$ siècle -, mais il se généralise. L'intervention prend la forme d'un consentement explicite ou de l'apposition du signum au bas de la charte, qui valait consentement. Cela étant, la gestion du patrimoine révèle aussi les rapports

25. H.-W. GoETZ, «Coutumes d'héritage et structures familiales au haut Moyen Âge», dans F. Bougard, C. La Rocca, R. Le JAN éd., Sauver son âme et se perpétuer. Transmission du patrimoine et mémoire dans le haut Moyen Âge, Rome, p. 203-237 (p. 222-223).

26. Sur la Bavière, voir G. BüHRER-THIERRY, «Femmes donatrices, femmes bénéficiaires. Les échanges entre époux en Bavière du VIII ${ }^{e}$ au $x^{e}$ siècle», dans Dots et douaires..., p. 329-351 (p. 338-341).

27. Die Urkunden der Arnulfinger, $\mathrm{n}^{\circ}$ 4, 5 (13 mai 706), 6 (2 mars 714).

28. Sur les précaires et leur diffusion, Les Transferts patrimoniaux en Europe occidentale, VIII ${ }^{e} X^{e}$ siècle, MEFRM 111-2 (1999).

29. Urkundenbuch zur Geschichte der mittelrheinischen Territorien [electronic resource http:// books.google.com], éd. H. Beyer, L. Eltester, A. Goerz, Coblence, 1860-1874, 1, nº 14. R. Le JAN, Famille..., p. 353. 
de force au sein même du couple, suscitant des conflits d'intérêts autour des héritages, des douaires, etc. En 1006, on enregistre le règlement d'un conflit entre le comte Thierri de Bar et son épouse Sonehilde qui contestait une donation de son mari faite sans son consentement ${ }^{30}$. Les biens provenaient probablement de son patrimoine, puisqu'elle était assistée par un de ses parents et par son fils, mais le procès révèle la redéfinition des droits de chacun des membres d'un consortium, qui comprend non seulement le couple mais aussi tous les ayants droit parentaux et féodaux ${ }^{31}$.

\section{Le couple, un acteur politique?}

L'exercice de la puissance publique, à savoir la justice et les affaires militaires, était lié au statut. Dans ce domaine, on a eu trop vite tendance à séparer les genres, car dans la Gaule mérovingienne ou la Germanie ottonienne, des régentes ont gouverné les royaumes, comme des hommes, au nom de leurs fils ou petits-fils mineurs.

Depuis l'époque mérovingienne, le couple royal était doté d'un statut, dès lors que l'épouse du roi portait le titre de regina, calqué sur celui de rex. La reine était dotée de biens fiscaux, de domaines royaux, auxquels se sont ajoutées à l'époque carolingienne des abbayes royales, puis des parts de revenus fiscaux (sur les ateliers monétaires par exemple). Cette dot initiale, formée de biens chargés d'une forte symbolique, augmentée à la naissance d'héritiers de cadeaux royaux, constituait pour elle l'assise à partir de laquelle elle pouvait exercer un pouvoir ${ }^{32}$. Quand la reine fut couronnée et ointe, au IX ${ }^{\mathrm{e}}$ siècle, ce statut s'affermit encore.

Cependant, toutes les épouses royales mérovingiennes n'ont pas été dotées et la polygynie a grandement limité la visibilité d'un certain nombre de couples royaux. La reine du $\mathrm{vI}^{\mathrm{e}}$ siècle est d'ailleurs perçue comme la regina du roi et Frédégonde est la seule reine dont nous savons qu'elle a exercé une réelle action politique, aux côtés de son mari Chilpéric, après avoir éliminé ses rivales. Les autres reines sont peu visibles du vivant de leur mari. Sur la scène internationale, où les alliances matrimoniales ont joué un rôle important au début du vi ${ }^{\mathrm{e}}$ siècle, à aucun moment les femmes n'ont été des actrices en tant qu'épouses, des médiatrices capables d'empêcher les guerres. Les rois règlent directement les affaires diplomatiques, en invoquant les liens de parenté nés des mariages, mais

30. Chronique et chartes de l'abbaye de Saint-Mihiel, éd. A. Lesort, Paris, 1909, nº 32; R. LE JAN, Famille..., p. 355.

31. R. LE J J , Famille..., p. 355-356.

32. R. LE JAN, «Douaires et pouvoirs des reines en Francie et en Germanie ( $\left(\mathrm{vl}^{\mathrm{e}}-\mathrm{X}^{\mathrm{e}} \mathrm{s}\right.$.)», dans EAD., Femmes, pouvoirs et société dans le haut Moyen Âge, Paris, 2001, p. 68-88. 
sans avoir recours à la médiation de celles par qui passait le lien ${ }^{33}$. Au VII ${ }^{\mathrm{e}}$ siècle, le statut du couple royal s'est affermi. Le couple royal est devenu plus visible, ce qui explique peut-être que nous ne connaissions qu'une seule épouse aux successeurs de Dagobert. La reine Bathilde a soutenu l'évêque de Lyon Genesius auprès de Clovis II, la reine Bilichilde a participé avec sa mère Chimnechilde et son mari Childéric II aux persécutions contre l'abbesse Vulfetrude à Nivelles, et on peut penser que les deux femmes étaient elles-mêmes les initiatrices de ces hostilités. La reine Chrodechilde fut assassinée avec son mari Thierri III en 690-691, comme si les nobles l'avaient aussi rendue responsable des outrages commis par le roi à leur encontre.

Le rôle public joué par Plectrude au début du viII' siècle s'inscrirait dans cette évolution. Elle a collaboré activement au renforcement du pouvoir pippinide en Austrasie, et soutenu directement la mission du missionnaire anglo-saxon Willibrord à qui elle a ouvert son réseau en Rhénanie, dans la région de Cologne, mais aussi en Thuringe, auprès du duc Heden II auquel elle était apparentée. En Neustrie, Plectrude est aussi aux côtés de son mari, elle participe avec ses fils à l'assemblée de Rouen et assiste à la cérémonie au cours de laquelle le monastère de Fleury-en-Vexin, restauré par le couple, est transféré aux moines de Fontenelle. En 708, quand Pépin II fut amené à revoir sa succession à la suite de la mort de son fils aîné Drogon, le couple écarta Charles, qui était pourtant majeur ${ }^{34}$. À la mort de Pépin II enfin, elle agit comme une véritable régente, jusqu'à ce que Charles Martel n'en vienne finalement à bout. En ce qui concerne Charles Martel lui-même, nous ne savons rien de l'action de Chrotrude, qu'il avait épousée avant la mort de son père, alors que nous sommes mieux renseignés sur la place de Swanahilde, la princesse bavaroise qu'il ramena de Bavière en 725 (du vivant de Chrotrude?). Apparentée aux ducs de Bavière et d'Alémanie, probablement liée à la famille de Plectrude, elle figure comme regina dans l'entrée de 818 où la famille carolingienne tout entière est inscrite sur le livre de Reichenau ${ }^{35}$. Quel qu'ait été son statut, elle sut s'imposer aux côtés du «prince» Charles. Elle l'a remplacé en son absence et a joué un véritable rôle politique à Paris, aux côtés du comte Gairefred ${ }^{36}$, exactement comme le fit plus tard la reine Fastrade quand elle rendit des jugements pendant que Charlemagne était occupé en Saxe. Swanahilde apparaît dans la dernière charte de Charles Martel en faveur de Saint-Denis,

33. R. LE J JN, «Mariage et relations internationales: 1'amitié en question? », dans Le relazioni internationazionali nell'alto medioevo, Atti delle settimane LVIII, Spoleto, 8-12 aprile 2010, Spolète, 2011, p. 188-224 (197-204).

34. B. Kasten, Königssöhne..., p. 65-66.

35. Confraternitates Augienses (désormais Cod. Aug.), dans Libri Confraternitatum Sancti Galli, Augiensis, Fabariensis, éd. P. PIPER, MGH Necrologia, Berlin, 1885 : II, p. 460.

36. P. Fouracre, The Age..., p. 161-164. 
en septembre 741, en tant qu'illustris matrona, un titre qu'avait porté Plectrude. Son influence fut assez grande pour obtenir de Charles qu'il accorde une part à leur fils Grifon, encore mineur, dans le partage de 740 entre ses fils Carloman et Pépin. L'influence de Bertrade, épouse de Pépin III, ne fut pas moins grande après la naissance de ses fils et jusqu'à la mort de son mari en $768^{37}$.

Cependant, le développement n'est pas linéaire, si l'on suit les sources en tout cas. À partir du règne de Louis le Pieux, on change d'échelle. Le couple impérial et ses enfants se mettent en scène dans de grandioses cérémonies qui reproduisent l'ordre et la hiérarchie, comme lors du sacre impérial de 816 ou du baptême des chefs danois en 826. La reine jouit désormais d'une autorité exceptionnelle, son statut est conforté, publicisé, car de son honestas dépend désormais celui du palais, si bien que le couple royal se trouve dès lors exposé à la calomnie ${ }^{38}$. En Italie, l'évolution est poussée à son terme quand, en 848, l'impératrice Ermengarde, épouse de Lothaire, est qualifiée de consors regni dans un diplôme royal ${ }^{39}$. La tradition est ensuite amplifiée par Engelberge, épouse de l'empereur Louis $\mathrm{II}^{40}$, qui est institutionnellement associée au pouvoir royal.

Les valeurs de coresponsabilité qui imprègnent les élites dirigeantes du royaume conduisent à une réflexion intense sur la place du couple dans la société. Dans les années 820, le comte d'Orléans, Matfrid, commande à l'évêque Jonas, principal théoricien de la ministérialité royale, un traité sur l'Institution des laïcs, qui est en grande partie consacré au mariage et aux responsabilités des époux. Pour autant, les couples aristocratiques du IX siècle n'apparaissent qu'en filigrane, et il faut attendre les années 880 pour qu'interviennent des changements importants, non seulement dans le mode de représentation de l'épouse, mais aussi dans la gestion du pouvoir politique. Pour les mesurer, il vaut la peine de comparer trois générations de femmes, entrées par mariage dans l'illustre famille des Guilhemides: Dhuoda, Ermengarde et Engelberge. De Dhuoda, épouse de Bernard de Septimanie, on ne sait rien sinon qu'elle a collaboré étroitement avec Bernard en gérant pour lui ses affaires de la Marche d'Espagne. En revanche,

37. Sur le rôle de Bertrade, voir J. L. Nelson, «Bertrada», dans M. BeCher et J. JARnut, Das Dynastiewechsel von 751, Münster, 2004, p. 93-108.

38. M. DE Jong, The Penitential State. Authority and Atonement in the Age of Louis the Pious, 814-840, Cambridge, 2009, p. 188-213. G. BüHRER-THIERRY, «La reine adultère », Cahiers de Civilisation médiévale, 35 (1992), p. 299-312.

39. Sur 1'usage juridique du terme, P. Delogu, « "Consors regni”. Un problema carolingo», Bolletino dell'Istituto storico per il medioevo e Archivio muratoriano, 76 (1964), p. 47-98.

40. Sur Engelberge, voir F. Bougard, art. Engelberga, dans Dizionario biografico degli Italiani, t. 47, Rome, 1993, p. 668-676, http://www.treccani.it/enciclopedia/imperatrice-engelberga (Dizionario-Biografico)/; C. LA RocCA, «La reine et ses liens avec les monastères dans le royaume d'Italie», dans R. LE JAN éd., La Royauté et les élites dans l'Europe carolingienne, Villeneuved'Ascq, 1998, p. 269-284 (p. 279-283). 
sa belle-fille Ermengarde, épouse de Bernard Plantevelue, joue un rôle public beaucoup plus affirmé. Sans doute ses origines royales l'y ont-elles conduite, ainsi que sa position d'héritière. Dans un acte de Brioude de 881 , une donation est faite par un certain Petrus pour les âmes de Bernard, gloriosissimus comes, de son successeur Bernard, eximii et praexecellentissimi comitis, et de son épouse Irmengarde, gratia Dei comitissa ${ }^{41}$. Le titre comitissa est calqué sur celui de la regina et se diffuse rapidement au $\mathrm{x}^{\mathrm{e}}$ siècle, traduisant un changement profond dans le mode de représentation des élites dirigeantes, lié à la conjugalisation des modèles familiaux et à la transmission directe des honneurs ${ }^{42}$. Pour Ermengarde, qui est gratia dei comitissa parce qu'elle a transmis à son époux les droits sur l'Auvergne, le statut se charge d'un fondement sacré qui lui donne un caractère institutionnel, comparable au queenship de la reine. Ermengarde intervient directement dans des affaires aussi éminemment politiques que les relations avec les grands de la principauté: selon la Vie de Géraud d'Aurillac, tandis que Bernard Plantevelue confiait à Géraud leur fils adolescent Guillaume comme nutritus, par amitié pour lui, Ermengarde, parce qu'elle l'aimait beaucoup, a cherché à faire épouser à ce même Géraud une de leurs filles ${ }^{43}$. Sa belle-fille Engelberge, épouse de Guillaume le Pieux, n'est pas en reste. Petite-fille de l'empereur Louis II et de l'impératrice Engelberge par sa mère Ermengarde, fille du roi Boson de Provence, elle était d'ascendance carolingienne et apparentée aux rois de Bourgogne. Son mariage ouvrit à Guillaume le Pieux le Lyonnais et, en janvier 917, Engelberge, comitissa, donna, par le biais de son mari Guillaume et de quatre autres fidéjusseurs, le fisc de Romans, dans le pagus de Lyon à Cluny, pour le salut de Guillaume et le sien, et pour celui de son frère Louis, de son père et de sa mère ${ }^{44}$. La fondation de Cluny, sur des terres qui semblent à première vue excentrées, aux confins de la Bourgogne rodolphienne et des terres bosonides, rentrait en fait dans une stratégie territoriale pensée par le couple. Il n'est donc pas étonnant que Guillaume ait décidé d'y associer son épouse, alors même que le domaine lui venait de sa sœur qui l'avait hérité de leur mère. Le couple était un acteur politique à part entière ${ }^{45}$.

Le champ d'action des épouses s'élargit pour se rapprocher de celui de leurs époux. Les reines accèdent ainsi, au x $x^{\mathrm{e}}$ siècle, au devant de la scène politique,

41. Cartulaire de Brioude, éd. H. DonIOL, Clermont-Ferrand, 1863, $\mathrm{n}^{\circ} 131$.

42. R. LE JAN, «L'épouse du comte au IX ${ }^{\mathrm{e}}$ siècle. Évolution d'un modèle et idéologie du pouvoir», dans EAD., Femmes..., p. 21-29.

43. Vita Geraldi I, c. 34 (PL 133, col. 663).

44. A. Bernard, A. Bruel éd., Recueil des chartes de l'abbaye de Cluny, 1, Paris, 1876, nº 205 , p. 193.

45. R. LE JAN, «La fondation de Cluny, le genre et le premier âge féodal», dans D. IoGNAPrat, F. Mazel, I. Rosé éd., Cluny, le monachisme et la société au premier âge féodal (880-1050), Rennes, 2013, p. 215-223. 
par une association plus étroite à l'exercice du pouvoir, qu'elles aient le titre de consors comme les reines d'Italie puis les impératrices, ou qu'elles ne l'aient pas comme les reines de Francie ou d'Angleterre ${ }^{46}$. On les voit défendre des forteresses, commander aux vassaux, mais aussi jouer le rôle d'ambassadrice. Les princesses ne sont pas en reste. Une cousine et contemporaine d'Engelberge, Berte de Toscane, fille du roi Lothaire II († 869) et mariée en secondes noces au marquis Adalbert II de Toscane, illustre ces capacités d'action. Du vivant même de son mari, en 905-906, elle envoie avec des cadeaux une lettre au calife abbaside de Bagdad, al-Muktafi, dans laquelle elle propose un pacte au calife après que sa flotte eut intercepté les navires arabes en Méditerranée et fait prisonnier le commandant eunuque de la flotte ${ }^{47}$. Entre le calife et l'empereur byzantin, Berte, qualifiée de «reine des Francs », se trouve au cœur d'un réseau dont elle apparaît comme un acteur direct. Liutprand de Crémone confirme par ailleurs que Berte, du vivant même de son mari, dirigeait la politique familiale ${ }^{48}$. Ce cas est exceptionnel, mais il est emblématique des changements intervenus à la fin du $\mathrm{IX}^{\mathrm{e}}$ siècle. Le consortium a été redéfini, l'épouse aristocratique est perçue et présentée comme une domina au côté du dominus. Cette autorité lui permet non seulement de conduire ses propres actions, mais aussi d'être directement impliquée dans les opérations politiques du consortium. Selon Adémar de Chabannes, tandis que Foulques Nerra s'emparait de son ennemi le comte du Maine Herbert, son épouse Hildegarde aurait tenté de s'emparer de la femme d'Herbert. Par crainte des représailles des gens et de l'épouse d'Herbert, Foulques aurait renoncé à tuer Herbert ${ }^{49}$. On charge aussi les comtesses de développer des actions de médiation politique et ce rôle médiateur s'affirme en particulier au moment de la réforme grégorienne, quand les femmes deviennent des médiatrices privilégiées entre laïques et ecclésiastiques ${ }^{50}$. Cependant, l'arbre ne doit pas cacher la forêt. La solidité du couple dépendait toujours autant de sa capacité à se reproduire, des rapports de force au sein de la famille, et des intérêts politiques en jeu.

46. R. LE JAN, «Douaires...», p. 76-88.

47. Sur cette lettre, G. GANDino, «Aspirare al regno : Berta di Toscana», dans C. LA Rocca éd., Agire da donna. Modelli e pratiche di rappresentazione (secoli VI-X), Turnhout, 2007, p. 249-268; T. LAZARRI, «La rapprezentatione dei legami di parentela e il ruolo delle donne nell'alta aristocrazia del regno italico (secc. IX-x) : l'esempio di Berta di Toscana», ibid., p. 129-149.

48. Liutprand de Crémone, Antapodosis II, 39 (éd. P. Chiesa, Turnhout, 1998, p. 51); T. LAZZARI, «La rappresentazione...», p. 139-140.

49. AdÉmar de Chabannes, Chronique III, c. 64 (trad. Y. Chauvin et G. Pon, Turnhout, 2003 , p. 284).

50. T. DE Hemptinne, «Women as Mediators between the Powers of comitatus and sacerdotium. Two Countesses of Flanders in the Eleventh and Twelfth Centuries», dans M. Gosman, A. VANDERJAGt et J. Veenstra éd., The Propagation of Power in the Medieval West, Groningue, 1997, p. 287-299. 
Le couple conjugal n'est pas une donnée sociale invariable et intangible, mais une construction soumise au changement. Au haut Moyen Âge, le système de parenté cognatique et la faiblesse des structures étatiques placent le mariage au centre des échanges qui assurent l'équilibre social et qui régulent la compétition entre les groupes aristocratiques, mais il faut distinguer mariage et couple. Au début de la période, les tendances polygyniques affaiblissent la visibilité du couple, quand le rapport de force ne permet pas à une épouse de s'imposer. À terme cependant, la sédentarisation et la christianisation ont joué en faveur du mariage légal en promouvant la conjugalité et la monogamie. Dès le viI siècle, des couples développent des stratégies patrimoniales et politiques, ce qui permet de promouvoir, au IX $\mathrm{I}^{\mathrm{e}}$ siècle, une idéologie sociale fondée sur le modèle conjugal. Cependant, c'est seulement à la fin du Ix ${ }^{\mathrm{e}}$ siècle qu'interviennent des changements profonds qui conduisent à associer directement l'épouse aristocratique à la gestion du pouvoir et à mettre en scène le couple en tant qu'acteur social et politique.

Régine LE J AN - Université Paris I Panthéon Sorbonne, Histoire, 1 rue VictorCousin, 75005 Paris

\section{Le couple aristocratique au haut Moyen Âge}

Le couple conjugal n'est pas une donnée sociale invariable et intangible, mais une construction soumise au changement. Au haut Moyen Âge, le système de parenté cognatique et la faiblesse des structures étatiques placent le mariage au centre des échanges qui assurent l'équilibre social et qui régulent la compétition entre les groupes aristocratiques, mais il faut distinguer mariage et couple. Au début de la période, les tendances polygyniques affaiblissent la visibilité du couple, si le rapport de force ne permet pas à une épouse de s'imposer. À terme cependant, la sédentarisation et la christianisation ont joué en faveur du mariage légal en promouvant la conjugalité et la monogamie. Dès le $\mathrm{VII}^{\mathrm{e}}$ siècle, des couples développent ensemble des stratégies patrimoniales et politiques, ce qui permet aux évêques de promouvoir, au IX ${ }^{\mathrm{e}}$ siècle, une idéologie sociale fondée sur le modèle conjugal. Cependant, c'est seulement à la fin du $\mathrm{IX}^{\mathrm{e}}$ siècle qu'interviennent des changements profonds qui conduisent à associer directement l'épouse aristocratique à la gestion du pouvoir et à mettre en scène le couple en tant qu'acteur social et politique.

couple - aristocratie - haut Moyen Âge - mariage - parenté 


\section{The Aristocratic Couple in the Early Middle Ages}

Conjugal couple is no invariable and inviolable social fact, but a changeable construction. In the Early Middle Ages, the cognatic Kinship system and the weakening of the State have placed the marriage in the middle of the social exchange system which assured the balance within the society and which regulated the competition between aristocratic groups. But we have to make the difference between marriage and couple. At the beginning of the period, polygenic trends weakened the conjugal visibility, if the balance of power didn't allow one of the spouses to get the upper hand upon the other. Nevertheless, afterward, people's settlement and Christianization have worked in favor of legal marriage, by promoting conjugality and monogamy. From the seventh century, couples have developed together patrimonial and political strategies, and in the ninth century, Carolingian bishops could promote a social ideology based on the conjugal model. Nevertheless, it's only at the end of the ninth century that deep change led to associate the aristocratic legal spouse directly to power management and to present the couple as a social and political actor.

couple - aristocracy - Early Middle Ages - marriage - kinship 